\title{
Cryo-FIB Milling Reveals Complex Vesicular Architecture in Photosynthetic Bacteria
}

\author{
Jade M. Noble ${ }^{1}$, J. Lubieniecki ${ }^{2}$, J. Plitzko ${ }^{2}$, H. Engelhardt ${ }^{2}$, W. Baumeister ${ }^{3}$, and L.F. Kourkoutis ${ }^{4,5}$
}

${ }^{1}$. Robert Frederick Smith School of Chemical and Biomolecular Engineering, Cornell University, Ithaca, New York, USA.

2. Dept. of Molecular Structural Biology, Max Planck Institute of Biochemistry, Martinsried, Germany.

${ }^{3 .}$ School of Applied and Engineering Physics, Cornell University, Ithaca, New York, USA.

4. Kavli Institute at Cornell for Nanoscale Science, Ithaca, New York, USA.

Photosynthesis by plants, algae, and photosynthetic bacteria is a significant source of the global energy supply. Photosynthetic vesicles, such as the chromatophore in Rhodobacter sphaeroides, are fascinating both fundamentally and technologically. Each chromatophore is capable of photosynthesis by absorbing sunlight as electronic excitation and converting it into chemical energy in form of ATP. Previous scientific inquiries prioritized characterizing the protein-pigment complexes on a chromatophore surface, and how they inform our understanding of photosynthesis by eukaryotic organelles, like chloroplasts. More recent work demonstrates new approaches to engineering biorenewable devices with chromatophore complexes as the active components, renewing interest in Rba. sphaeroides. Despite significant advances in understanding how proteins interact on the surface of a single vesicle, decadeslong deliberation on how chromatophores in Rba. sphaeroides interact with neighboring vesicles continues today. As the reticulated nature of chromatophores may have a significant impact on the photosynthetic mechanism in Rba. sphaeroides and biorenewable technologies, it is imperative to investigate the extent of connectivity between vesicles throughout the bacteria both in situ and in 3D.

Understanding chromatophore connectivity is hampered by limitations of techniques typically used to assess their structure. For instance, atomic force microscopy has been used to image the arrangement of proteins on the surface of chromatophores, but isolation of vesicles from the bacteria is required and can disturb their network. Transmission electron microscopy (TEM) provides visualization of peripheral structure in Rba. sphaeroides with nanoscale detail, but resolving the internal structure is often hampered by cellular thickness. The conventional thinning technique is sectioning by cryoultramicrotomy, which can introduce compression artifacts. Most of these experimental limitations can be avoided with alternative techniques such as cryo-focused ion beam milling (cryo-FIB) and cryoelectron tomography (cryo-ET). Sample fixation by vitrification preserves the sample in a near-native, liquid-hydrated state avoiding staining artifacts. Cryo-FIB milling thins a specimen with a collimated beam of $\mathrm{Ga}^{+}$ions, providing site-specific, ultra-thin sections without the artifacts associated with cryoultramicrotomy [1-2]. Furthermore, thinning occurs directly on the TEM grid where specimens have been previously vitrified, reducing the risk of contamination. Cryo-FIB is a robust technique that has been used with cryo-ET to visualize both bacteria and photosynthetic organelles in 3D [3-4].

In this study, we use an on-grid cryo-FIB preparation of fully-hydrated, vitrified Rba. sphaeroides to gain an unprecedented view of the internal structure of the bacteria in its native state (Fig. 1 B,C). We describe the macromolecular landscape of Rba. sphaeroides, including vesicular biogenesis, development, organization, and connectivity (Fig. 2 A,B). This study provides an assessment of the diversity of vesicular architecture that was inaccessible to previous experimental techniques, as well as the biophysical motivation for connectivity between vesicles [5]. 


\section{References}

[1] A Al-Amoudi, LPO Norlen and J Dubochet, J. Struct. Biol. 148 (2004), p. 131-135.

[2] A Rigort et al, Proc. Natl. Acad. Sci. USA 109 (2012), p. 4449-4454.

[3] M Marko et al, Nat. Methods 4 (2007), p. 215-217.

[4] Engel et al, eLife 4 (2015), p. e048.

[5] This work was supported in part by the Packard Foundation.
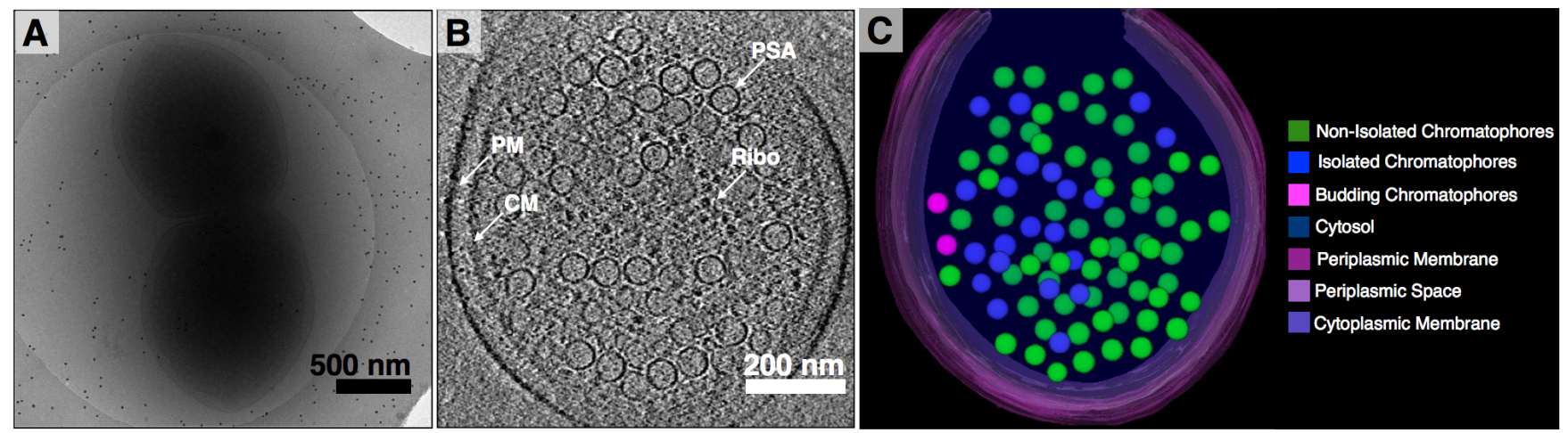

Figure 1. The cytoplasmic space of whole Rba. sphaeroides bacteria (A) is occluded to the electron beam due to sample thickness. An orthoslice of an Rba. sphaeroides bacteria thinned by cryo-FIB milling (B) reveals the entire cytoplasmic architecture with nanoscale resolution, including the periplasmic membrane (PM), cytoplasmic membrane (CM), photosynthetic apparatuses (chromatophores; PSA), and prokaryotic ribosomes (Ribo). Segmentation and tomographic rendering of chromatophores display a diversity of architecture, including budding from the cytoplasmic membrane and connectivity between neighboring vesicles (C).

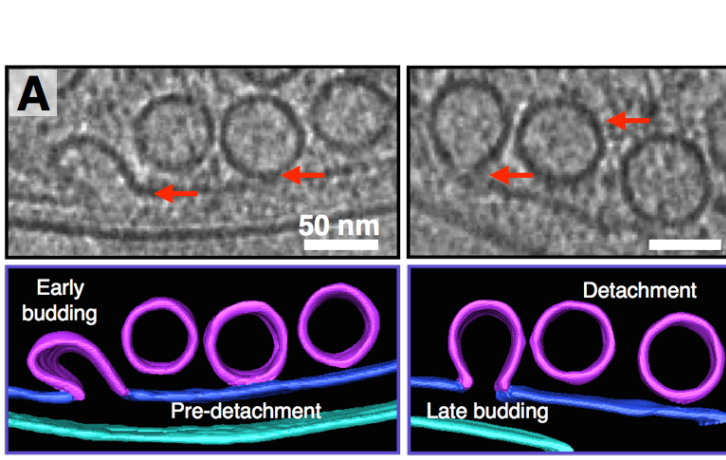

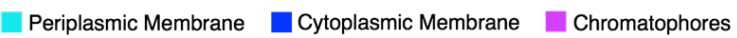

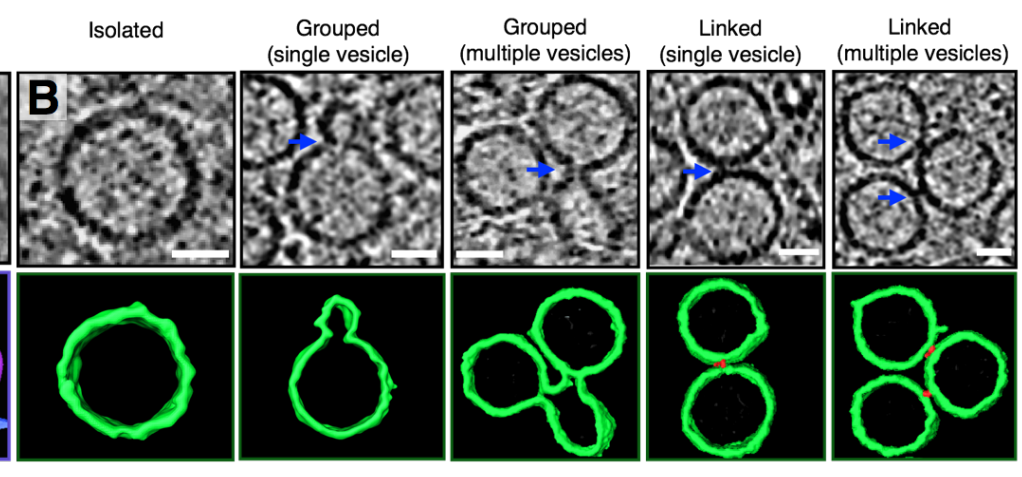

Chromatophores

- Linkers

Figure 2. Tomographic data from Rba. sphaeroides prepared by cryo-FIB capture a montage of vesicular biogenesis (A). Each stage (early budding, late budding, pre-detachment and detachment) is marked by differences in vesicular shape and the degree of continuity with the CM (scale bars: $50 \mathrm{~nm}$ ). Centermost vesicles, accessible only by cryo-FIB, show rich architecture (B). Although some vesicles appear to be isolated, most are connected to neighboring vesicles via secondary budding events or small linkers that connect fully matured chromatophores to one another (scale bars: $25 \mathrm{~nm}$ ). 